\title{
Functional Design on Purchase Quotation System for Machine Element
}

\author{
Tong Ling ${ }^{1}$, Li Linlin ${ }^{1}$, Zhang Rui ${ }^{1}$, Liu Lei ${ }^{2}$ \\ ${ }^{1}$ No.208 Research Institute of China Ordnance Industries, Beijing 102202 \\ ${ }^{2}$ College of Engineering, Bohai University, Jinzhou, 121013
}

Keywords: machine element; purchase quotation system; functional design; functional structure model; optimal purchase quantity model

\begin{abstract}
There are many different kinds of machine element, with professional development of industrial production, only a few machine elements needed by factories are for their own production, most are needed to be purchased. Using purchase quotation system for machine element is an effective mean to ensure the supply of elements and cost savings. This paper designs system functions to provide technical support for system development. Based on optimal purchase quantity model and functional requirement analysis, system design constructs functional structure model, including "Purchase model maintenance, Element category maintenance, Element information management, Purchase plan management, Supplier quotation management, Supplier information management, Data comprehensive report, Software configuration management" modules. Based on the results of this paper, we can develop a full-featured, affordable information system.
\end{abstract}

\section{Introduction}

The basic element of machine is called "Machine element", also known as machine part, which is the inextricably individual element composed of machinery and machine, and it is the basic unit of machine. As a discipline, starting from the overall mechanical design, machine element uses the outcomes of the relevant disciplines to research principle, structure, characteristics, application, failure models, load capacity and design program of various basic element; research theory, method and guideline of basic element design, and thus establish theoretical system of the discipline combined with the reality, which has become an important basis for researching and designing machine. With the development of the machine industry, due to the emergence of the new design theory and methods, new materials and new technology, machine element enters into a new development stage. Better achieve the comprehensive of variety of disciplines, achieve the combination of macro and micro, explore new principles and structures, use dynamic design and accurate design, use computer more effectively, further develop design theory and method, are the important trend of the discipline development.

Due to a wide variety of machine elements and the professional development of industrial production, only a few machine elements needed by factories are for their own production, most are needed to be purchased. The data of elements management belongs to dynamic information, not only has big data volume, but also the content is cumbersome, and therefore must ensure that the data is accurate. The development of information technology provides a good support for the development of machine element procurement and quotation. This paper designs procurement quotation system functions for machine element to provide technical support for system development.

\section{Optimal Purchase Quantity Model}

Optimal purchase quantity model also known as entire batch interval purchase model, quantity applies to the entire batch interval purchase, and not allows storage problem, that demand of some kind of machine elements per unit time is $D$, the inventory is gradually decreasing by consumed quantity per unit time, after a period of time, the inventory is down to zero, then begin to purchase 
and then arrive, the inventory increases from zero to maximum, then start next storage cycle, form multi-cycle storage model. Classic inventory curve is shown in Fig. 1.

This model has the following characteristics: not allow stockout or damage of stockout is infinity; when the inventory drops to 0 , supplement can be obtained immediately; the need is continuous and uniform; the unit storage cost is unchanged. Making [1-3]:

$D$ : demand rate, the demand for some kind of machine elements per unit time;

$Q$ : purchase quantity, the number of some kind of machine elements in a purchase;

$t$ : purchase interval, representing the time interval between two purchases.

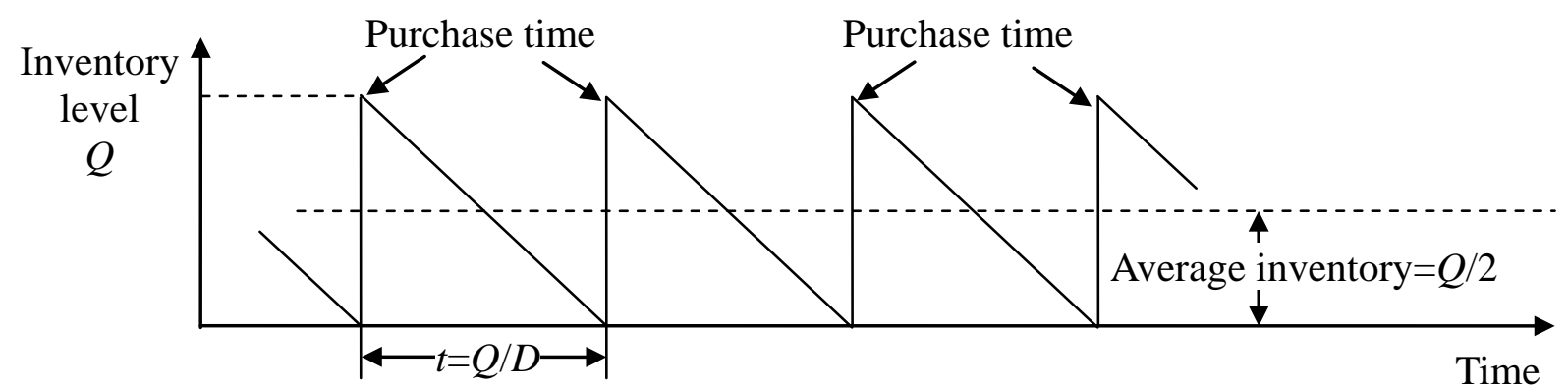

Fig. 1. Inventory level curve on optimal purchase quantity model

In one cycle, the maximum inventory is $Q$, the minimum inventory is 0 , and the demand is continuous and uniform, the average inventory in a cycle is $\frac{1}{2} Q$. The average inventory cost is $C H F$, storage cost is $C_{p}$, then:

$$
C H F=\frac{1}{2} C_{P} Q
$$

Since the first time, purchases is $Q$, after $t$ time, the inventory is 0 , the demand is $D$ and it continuous and uniform changes, therefore, the relation of purchases, demands and purchase cycle can be got, $t=\frac{Q}{D}$. Setting each purchase cost is $C_{D}$, the average purchase cost is $D H F$, and then you can get:

$$
D H F=\frac{C_{D}}{t}=\frac{C_{D} D}{Q}
$$

Setting the average total cost of stock is $T C(Q)$, and the average total cost of stock is equal to the sum of the average inventory cost and the average purchase cost, then:

$$
T C(Q)=\frac{1}{2} C_{P} Q+\frac{C_{D} D}{Q}
$$

Optimal value of purchases can be derived from $T C(Q)$ about the minimum of $Q$. Suppose $Q$ is continuous, the necessary condition for finding the optimal value of $Q$ is:

$$
\frac{d T C(Q)}{d Q}=\frac{1}{2} C_{P}-\frac{C_{D} D}{Q^{2}}=0
$$

You can get:

$$
Q^{*}=\sqrt{\frac{2 C_{D} D}{C_{P}}}
$$

Due to $\frac{d^{2} T C\left(Q^{*}\right)}{d Q^{2}}=\frac{2 C_{D} D}{Q^{* 3}}>0$, so $Q^{*}$ is the minimum of cost function $T C(Q)$, the optimal inventory cost is:

$$
T C^{*}=\frac{1}{2} C_{P} Q^{*}+\frac{C_{D} D}{Q^{*}}=\sqrt{2 C_{D} C_{P} D}
$$

Therefore, the optimal storage strategy of the optimal purchase quantity storage model is to purchase every $t$ time units:

$$
t^{*}=\frac{Q^{*}}{D}
$$


Purchase quantity is $Q^{*}$. The optimal inventory total cost of per unit time is $\sqrt{2 C_{D} C_{P} D}$ unit costs.

\section{Functional Requirements Analysis}

Functional requirement is the most important content of requirement analysis. Functional analysis is based on software objective to form results described by software functional model, and then quantitative or qualitative describe the proposed functional requirements to software, the main functional requirements of the system are as follows:

(1) Element information management [4]. Element is the basic constituent element of product, element management is standardized managing elements firstly. Element standardization, staring from product design ideas, around common elements, multifunction modules, standardized interface, common fixtures, geometric dimensioning and standard technology to manage. Using regularity of elements' structure shape and size distribution to classify and code machine elements according to the similarity of elements functions; the main elements should be classified according to the similarity of function and structure to form element family; tree-like hierarchical structure is used to express the classification elements to systematic manage elements; analyzing the geometric characteristics and features of elements, and all of its characteristic parameters are formed affair feature list.

(2) Purchase plan management. Purchase plan is making predictability arrangement and deployment for machine elements purchase plan management activities in the planning periods based on that enterprises managers understand the market supply and demand situation and understand the process of production activities and the laws on the use of machine elements. Purchase plan management is made based on the plan of production department or other departments, including purchase category, purchase quantity, date and so on. Make purchase plan management can effectively avoid risks and reduce losses to provide a basis for enterprises purchase; it is conducive to the rational allocation of resources to achieve the best economic benefits [5].

(3) Supplier quotation management. In order to standardize the purchase quotation process for machine element, establish an open, fair and just price environment and form a transparent pricing mechanism, it is essential to manage vendors' quotation. For each element, after market researches, the purchasing department should put forward a list included 5-10 candidate suppliers; enterprise should set up supplier selection team consisting of purchase, quality control, technical departments; and then the team calculates the total score to choose by scoring each candidate supplier. Suppliers quote their products on the Internet, and enterprise determines the final supplier after screening, final through the negotiation to determine final supply prices of machine elements.

(4) Supplier information management. Supplier management is an important function of purchase quotation management system. Supplier management module is centering supplier information management, standardized purchase process and advanced management idea for the purpose, from the basic information of suppliers, organization structure information, contact information, legal information, financial information and qualification information to investigate suppliers strength, then through managing the supply capacity, transactions, performance and other information to optimize the management and reduce costs [6,7]. The key step of supplier management is to find the best suppliers quickly, and attach complete transactions to support business decisions.

(5) Data comprehensive report. Report is to use tables and graphs to display data dynamically. The main features of computer-implemented reports is that data is dynamic and format is diverse, and report data and report format is separated completely, the user can only modify the data, or only modify format. Report design idea of the system is to determine the type and format of the report firstly, and then develop a report module to generate and print all reports. There is no uniform classification standard for report types, and the system divides report into detail table, summary table, analysis table and history table from the content. 
(6) Software configuration management [6]. Software configuration management (SCM) is a logo, organize and control revise technology. SCM is applied throughout the software process. Change is inevitable when the software is established, and the change exacerbates the confusion of developers in software development. The objective of SCM activity is to identify change, control change, ensure change implemented correctly and report the change to other relevant personnel. In some ways, SCM is a logo, organize and control revise technology, the purpose of it is to reduce errors to the minimum and increase productivity.

\section{Functional Structure Model}

Functional structure model is used to describe the hierarchical structure of the software functions. A rectangular box represents a module in structure chart, the line between the rectangular boxes represents call relation, and rectangular box located in the upper represents the module, it can call the module represented by rectangular box located in the below. The modular design should follow abstract, stepwise refinement, modularity and information hiding principles. The modular design of the software has different levels of abstraction. At the highest level of abstraction, you can use the language that the problem in which environment to describe method. While at the lower level of abstraction, the process method is used; stepwise refinement is a top-down design strategy, according to a top-down approach, software architecture refines the details of process and data for each level, until you can use the statements of programming language to achieve; the module can be separated in logic in program, modularity refers to "the degree of dispersion of that several discrete components consists of software, namely the degree of software modularity"; information hiding refers to that the implementation details of each module is hidden to other modules. Module function requirements analysis, modular design result is shown in Fig. 2. 


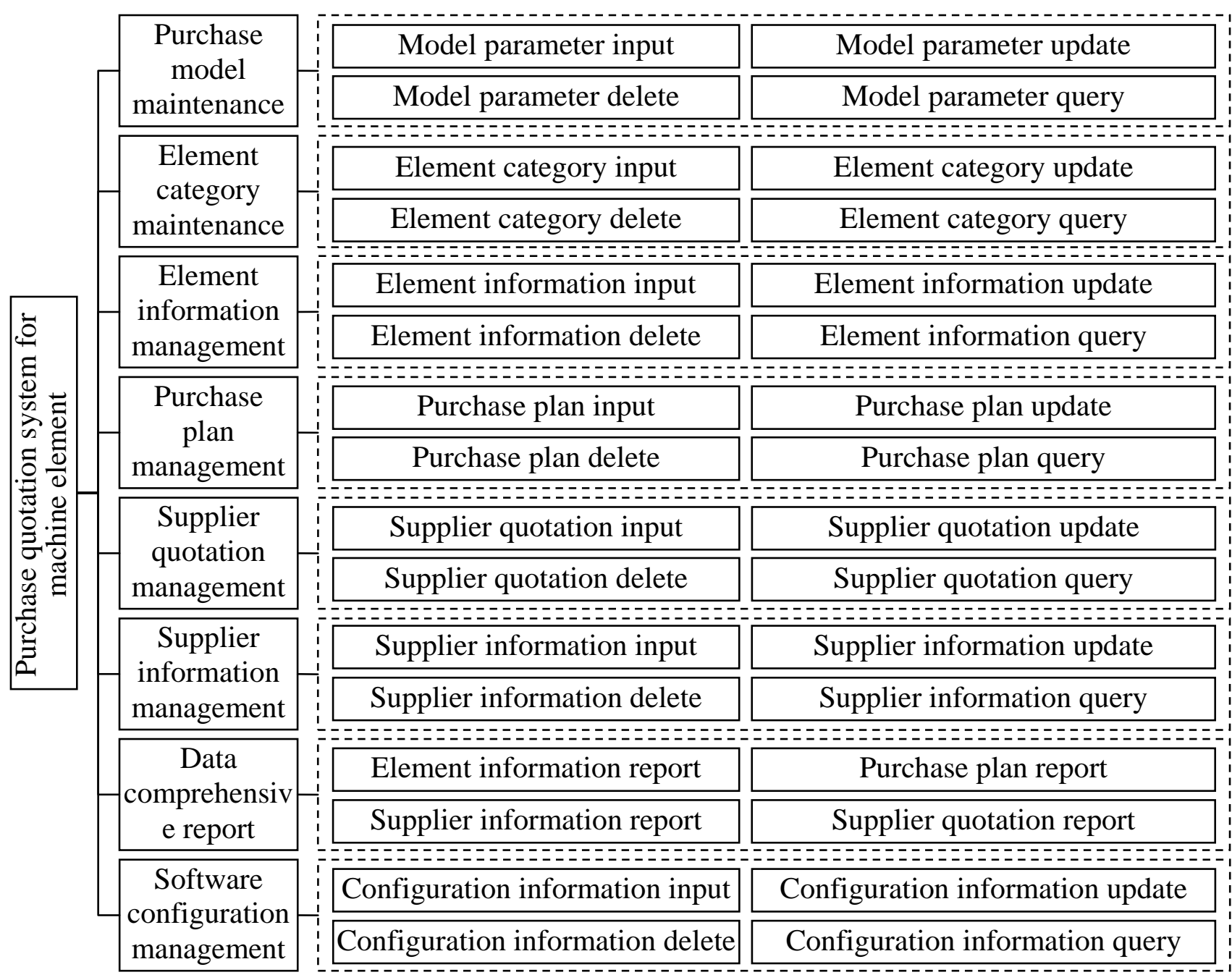

Fig. 2. Functional structure model on purchase quotation system for machine element

In the functional structure model shown in Fig. 2, in addition to comprehensive data reporting module, other modules include four types of database operations functions, namely "insert, modify, delete, query". The traditional database operation method is SQL statement, too many SQL statements are difficult to write and maintain. In order to simplify data manipulation, the system designs a data manipulation class packaged "insert, modify, delete, query" operations, which provides a very simple interface to complete the data operation by overloading. There is no need to write specific SQL statements when developing, so it reduces the amount of program code, reduces development time, improves program reliability and reduces maintenance workload. The main methods of data manipulation class are shown in Table 1.

Table 1. Main methods on data operating classes

\begin{tabular}{cccc}
\hline No & Class name & Operating & Description \\
\hline 1 & OpenConnection & Connection & Open database connection \\
2 & InsertRecord & Insert & Insert 1 records into the table \\
3 & UpdateRecord & Update & Update one or more fields in the table \\
4 & DeleteRecord & Delete & Delete records according to the condition \\
5 & QueryRecord & Query & Query records according to the condition \\
\hline
\end{tabular}

\section{Conclusion}

With China's rapid economic growth, commercial import and export values of China's industrial automation is increasing year by year, the management for machine element purchase quotation 
needs more convenient and practical management software. Element purchase quotation needs real-time acquisition, and promptly transmits all kinds of information to the related various departments according to needs, which has an important role for the supply of elements, sales and service, quality control, cost accounting and so on. Application of management software and computer network technology in line with industry provides a reliable guarantee to achieve the above functions, and it also provides users with a more open, more efficient, more standardized and better service. Software functions are the efficacy and role that software should have, the target of software is expressed and implemented by software functions, the software functions are the direct effect rendering to the users. Users understand, use and evaluate system through the software functions, and use software functions to perform work. Results of this study lay the foundation for developing full-featured, affordable purchase quotation system for machine element.

\section{References}

[1] X. Jiang, Z. X. Shan, "An economic order quantity model for inventory decision making," Finance and Accounting Monthly, vol. 36, no. 3, pp. 69-74, 2015.

[2] B. Luo, L. Feng, X. Q. Chen, "Economic order quantity model for off price merchandise with demand depends on selling price and stock under cross-selling," Systems Engineering-Theory \& Practice, vol. 34, no. 4, pp. 826-835, 2014.

[3] G. P. Zhou, "Design method of inventory economic order quantity model based on computer financial management," Finance Theory and Teaching, vol. 31, no. 4, pp. 72-75, 2013.

[4] X. J. Li, N. Lin, "Design of computer management system for mechanical parts," Software, vol. 35, no. 1, pp. 147-148, 2014.

[5] H. Sha, J. Ji, D. Wang, et al, "The design and application of medical equipment purchase plan management software," China Medical Equipment, vol. 12, no. 11, pp. 52-54, 2015.

[6] Q. C. Zou, "Development of Supplier Management Information System Based on Grading Mechanism," Logistics Technology, vol. 33, no. 7, pp. 432-434, 2014.

[7] H. Y. Sun, "Design of supplier management information system," Technological Development of Enterprise, vol. 33, no. 4, pp. 25-27, 2014.

[8] B. Chen, "Application of software configuration management in software development platform," Information \& Communications, vol. 28, no. 12, pp. 128, 2014. 